\title{
Clinical and Radiological Comparison between Three Different Minimally Invasive Surgical Fusion Techniques for Single-Level Lumbar Isthmic and Degenerative Spondylolisthesis: Minimally Invasive Surgical Posterolateral Fusion versus Minimally Invasive Surgical Transforaminal Lumbar Interbody Fusion versus Midline Lumbar Fusion
}

\author{
Mohamed Elmekaty ${ }^{1,2,3}$, Yoshihisa Kotani ${ }^{1}$, Emad El Mehy ${ }^{2}$, \\ Yohan Robinson $^{3}$, Ahmed El Tantawy ${ }^{2}$, Ivan Sekiguchi ${ }^{1}$, Ryo Fujita $^{1}$ \\ ${ }^{1}$ Spine and Spinal Cord Center, Steel Memorial Muroran Hospital, Muroran, Japan \\ ${ }^{2}$ Department of Orthopedic Surgery, Faculty of Medicine, Tanta University, Tanta, Egypt \\ ${ }^{3}$ Department of Surgical Sciences, Uppsala University Hospital, Uppsala, Sweden
}

Study Design: Retrospective cohort study.

Purpose: Comparison between three different minimally invasive surgical (MIS) fusion techniques for single-level lumbar spondylolisthesis.

Overview of Literature: There has been an increase in the development and utilization of MIS techniques for lumbar spine fusion. No study has compared the efficacy of MIS-posterolateral fusion (MIS-PLF), MIS-transforaminal lumbar interbody fusion (MIS-TLIF), and midline lumbar fusion (MIDLF) with modified cortical bone trajectory screws for lumbar spondylolisthesis.

Methods: Fifty-nine patients with single-level lumbar spondylolisthesis and a minimum follow-up period of 1 year were included in this study. The MIS-PLF, MIS-TLIF, and MIDLF groups included 22, 15, and 22 patients, respectively. The average age of the groups was 70.6, 49.3, and 62.7 years, respectively. The evaluation parameters were operation time, intraoperative bleeding, serum C-reactive protein (CRP) value, creatine kinase (CK) value, and overall functional outcome as per the Japanese Orthopedic Association Back Pain Evaluation Questionnaire (JOABPEO) score. The changes in the lumbar lordosis angle (LLA), segmental disc angle (SDA), and disc height were measured. Fusion rate, screw loosening, and loss of correction were also assessed.

Results: MIDLF showed a significantly shorter operation time (111 min), less bleeding amount (112.5 mL), and lower values of CRP and CK than the other two techniques. There was no significant difference in the JOABPEO scores of the three groups. MIDLF resulted in a greater increase in the LLA and SDA postoperatively. MIDLF and MIS-TLIF resulted in a significant increase in the middle disc height compared with MIS-PLF. MIDLF showed a lower loss of correction after 6 months postoperatively (2.6\%) than MIS-PLF (5.2\%) and MIS-TLIF (4.2\%). The fusion rate was $100 \%$ in the MIDLF and MIS-TLIF groups and $90 \%$ in the MIS-PLF group. Screw loosening occurred in $10 \%$ of the MIS-PLF cases, $7.14 \%$ of the MIS-TLIF cases, and $4.76 \%$ of the MIDLF cases.

Conclusions: MIDLF was the least invasive, and there was no significant difference between the three groups in terms of fusion, screw loosening, and clinical outcomes.

Keywords: Thoracolumbar spine; Isthmic and degenerative spondylolisthesis; Minimally invasive spine surgery; Midline lumbar fusion; Modified cortical bone trajectory screw

Received Nov 8, 2017; Revised Feb 4, 2018; Accepted Mar 4, 2018

Corresponding author: Mohamed Elmekaty

Department of Surgical Sciences, Uppsala University Hospital, Ing 61, 6tr, 75229 Uppsala, Sweden

Tel: +46-739611445, Fax: +46-18-509427, E-mail:melmekaty@yahoo.com 


\section{Introduction}

Lumbar spine fusion is widely considered to be the best surgical strategy for the management of different symptomatic spinal pathologies, particularly cases with biomechanical instability and deformity $[1,2]$. To obtain satisfactory results, lumbar spine fusion should achieve stable spinal segments to eliminate any further degenerative changes and provide decompression of the neural elements. Different techniques and approaches have been reported to accomplish spinal fusion. However, each method is technically demanding and involves its specific instrumentation, eligibility, and drawbacks [3-5].

Recently, attention has been paid to minimally invasive spinal surgery (MISS) as a successful, leading treatment choice compared with open conventional methods for the management of different spinal disorders [6-8]. This is attributable to the rapid development of intraoperative imaging systems and the production of novel spinal instrumentation and fusion devices in addition to modern retraction equipment. Consequently, newer methods of MISS are being established, and the scope of its effectiveness has increased to include patients with deformity, trauma, spondylolisthesis, and even tumor. The basic aim of MISS is to limit surgical incision and soft tissue damage that is usually associated with open conventional methods [8-10]. Therefore, MISS achieves superior results in terms of lower postoperative back pain, intraoperative bleeding amount, and postoperative hospitalization period, with improved quality of life. MISS techniques involve a learning curve, and once the surgeon has achieved expertise in MISS principles, the incidence of the initial complications and consumption of time reduce $[11,12]$.
Surgical management of lumbar spondylolisthesis can only be performed via decompression of the neural elements, posterolateral fusion (PLF), and transforaminal lumbar interbody fusion (TLIF) [13]. Minimally invasive surgical (MIS)-PLF is performed through a limited skin incision and percutaneous instrumentation, providing better overall outcomes than conventional PLF [14]. TLIF is considered one of the best methods of interbody arthrodesis because it spares one facet joint, which provides more stability of the spinal segment and facilitates future revision surgeries. However, open conventional TLIF is injurious for the back muscles and soft tissue, making the overall results unsatisfactory $[15,16]$. Owing to the use of percutaneous screws and the rod assembly system and the advances in the retraction instruments, MIS-TLIF has minimized the limitations of the open conventional method by decreasing intraoperative bleeding, soft tissue damage, postoperative pain, and hospital stay $[17,18]$.

Midline lumbar fusion (MIDLF) is a novel MIS fusion technique through which interbody fusion can be achieved via limited posterior midline incision and posterior instrumentation using novel cortical bone trajectory (CBT) screws [19].

The original CBT screw is inserted in the cephalic and divergent orientation, opposite to the traditional pedicular screw's trajectory (Fig. 1). This new trajectory allows the pedicular screws to engage the cortical bone of the dorsal lamina and the pedicle, enhancing biomechanical stability and the pullout strength of the CBT screws and decreasing the incidence of screw loosening $[19,20]$. We modified CBT (mCBT) to use larger and longer screws through variable insertion points to achieve the benefit of anterior load sharing (Fig. 2).

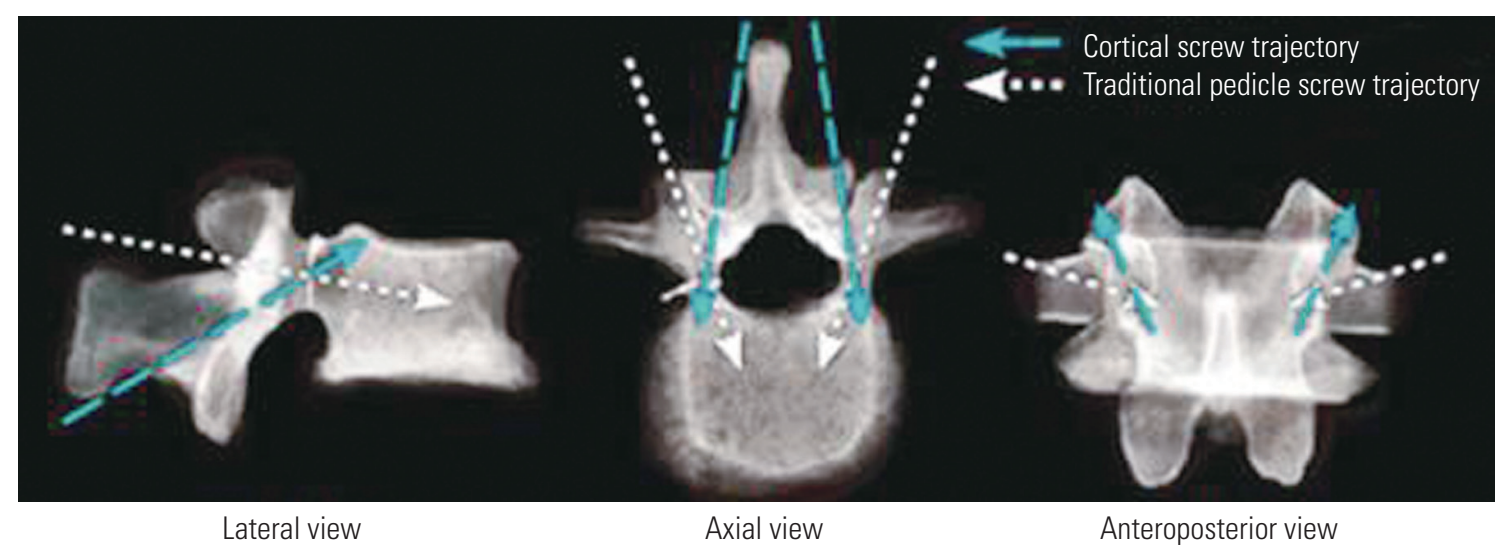

Fig. 1. Comparison of the cortical bone screw trajectory with the traditional pedicle screw trajectory. 
To the best of our knowledge, this is the first study to compare these three different MIS techniques in the management of single-level lumbar spondylolisthesis. We retrospectively compared the clinical and radiological outcomes of MIS-PLF, MIS-TLIF, and MIDLF.

\section{Materials and Methods}

\section{Patient demographics}

From December 2011 to May 2015, 59 patients (25 men and 34 women) underwent single-level MIS fusion for

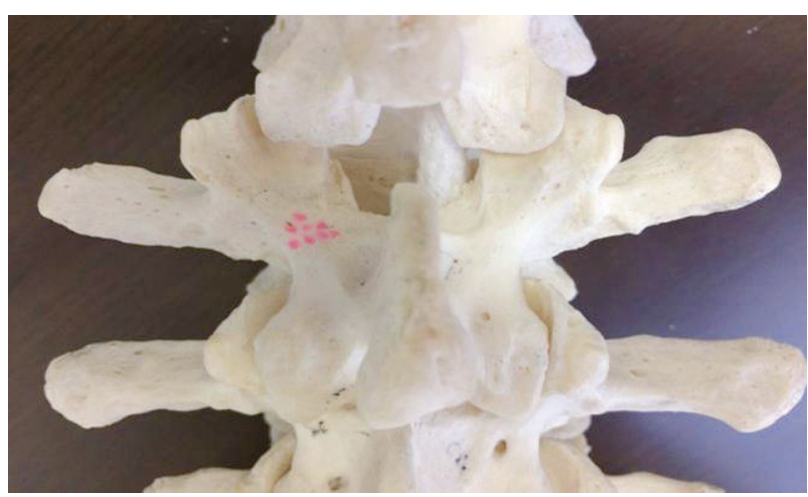

Fig. 2. Variable insertion points for the modified cortical bone trajectory screw (pink dots). lumbar isthmic spondylolisthesis (IS) (16 patients) and degenerative spondylolisthesis (DS) (43 patients). Of all the patients, 22 underwent MIS-PLF (four patients with DS had lumbar canal stenosis, and decompression was performed with fusion), 15 underwent MIS-TLIF, and 22 underwent MIDLF. The minimum follow-up period was 1 year. All the MIS surgeries were performed by the same surgeon, whereas the type of operation performed for each patient was determined according to operator and patient preferences. The mean age of the patients was $70.6 \pm 9.1$ years for the MIS-PLF group, $49.3 \pm 23.5$ years for the MIS-TLIF group, and $62.7 \pm 16.2$ years for the MIDLF group. The mean body mass index (BMI) was $23.4 \pm 4.2$, $23.7 \pm 3.6$, and $25 \pm 4.6 \mathrm{~kg} / \mathrm{m}^{2}$ for the MIS-PLF, MIS-TLIF, and MIDLF groups, respectively. The level of fusion was L1-L2 and L2-L3 in one patient, L4-L5 in 35, and L5-S1 in 22. The patients' demographic characteristics and clinical data are presented in Table 1.

\section{Patient selection}

The study subjects were operated on using single-level MIS-PLF, MIS-TLIF, or MIDLF for grades I and II IS or DS, according to the Meyerding method. Surgical intervention was considered after the failure of conservative treatment for at least 6 months. Patients with multi-level

Table 1. Patients' demographic characteristics and clinical data

\begin{tabular}{|c|c|c|c|c|}
\hline Characteristic & MIS-posterolateral fusion & $\begin{array}{l}\text { MIS-transforaminal } \\
\text { lumbar interbody fusion }\end{array}$ & $\begin{array}{l}\text { Midline lumbar } \\
\text { fusion }\end{array}$ & $p$-value \\
\hline No. of cases & 22 & 15 & 22 & \\
\hline Gender & & & & 0.4 \\
\hline Male & 10 & 8 & 7 & \\
\hline Female & 12 & 7 & 15 & \\
\hline Age $(y r)$ & $70.6 \pm 9.1$ & $49.3 \pm 23.5^{*}$ & $62.7 \pm 16.2$ & 0.0012 \\
\hline Body mass index $\left(\mathrm{kg} / \mathrm{m}^{2}\right)$ & $23.4 \pm 4.2$ & $23.7 \pm 3.6$ & $25.2 \pm 4.6$ & 0.43 \\
\hline Pathology & & & & 0.000011 \\
\hline Degenerative spondylolisthesis & 21 (4 cases with lumbar canal stenosis) & 4 & 18 & \\
\hline Isthmic spondylolisthesis & 1 & 11 & 4 & \\
\hline Vertebral body translation (Meyerding) & & & & 0.88 \\
\hline Grade I & 20 & 13 & 19 & \\
\hline Grade II & 2 & 2 & 3 & \\
\hline Average follow-up period (mo) & 22 & 18 & 14 & \\
\hline
\end{tabular}

Values are presented as number or mean \pm standard deviation. MIS, minimally invasive surgical. " $p<0.05$. 
fusion; grades III, IV, and V IS or DS; severe osteoporosis; BMI $>40 \mathrm{~kg} / \mathrm{m}^{2}$; active infection; or malignancy in the spinal region were excluded.

\section{Clinical evaluation}

All the patients were assessed for the operation time and intraoperative bleeding amount. Serum C-reactive protein (CRP) and creatine kinase (CK) were evaluated on postoperative day 1 (POD1) and postoperative day 7 (POD7) to compare the invasiveness and soft tissue affection of each procedure. The overall functional outcome was assessed using the Japanese orthopedic association back pain evaluation questionnaire (JOABPEQ) score, low back pain was assessed using the Visual Analog Scale (VAS) score, and leg pain was determined using the Oswestry Disability Index (ODI) before and at the 1-year follow-up. The incidence of surgical complications was also reported.

\section{Radiological evaluation}

We measured the changes in the lumbar lordosis angle (LLA), segmental disc angle (SDA), as well as the anterior, middle, and posterior disc heights (DHs) postoperatively using lateral plain radiography in a neutral position. Moreover, the percentage loss of correction was assessed by measuring the difference between the percent of slippage immediately postoperatively and 6 months thereafter. Fusion rates and screw loosening was assessed at 1 year postoperatively. Fusion was achieved when there was a solid fusion mass in the interbody space or posterolaterally in the absence of local instability (motion angle $<2^{\circ}$ on dynamic lateral radiographs) or implant failure. Computed tomography (CT) was performed to confirm the fusion status when it was doubtful.

\section{Surgical technique}

MIS-PLF was performed through either single midline or para-midline incisions using the mini open-Wiltse approach wherein the surgical plane was between the multifidus and longissimus muscles. Thereafter, using a selfilluminating expandable retractor, we decorticated the lamina, facet joints, and medial part of the transverse process. Then, percutaneous pedicle screws were inserted and assembled with rods. A bone graft was applied bilaterally to the decorticated posterolateral gutter (Fig. 3).

MIS-TLIF was performed through the mini-open Wiltse approach by removing the facet joint on the affected side. The disc space was prepared before inserting a single polyetheretherketone (PEEK) TLIF cage packed with autologous bone graft harvested from the posterior resected lamina and facets mixed with hydroxyapatite granules. Finally, pedicular screws were inserted directly via the miniopen Wiltse approach on the ipsilateral side and percutaneously on the contralateral side and assembled with rods (Fig. 4).

MIDLF includes TLIF and posterior instrumentation using novel mCBT screws with intraoperative $\mathrm{CT}$ (O-arm) image-guided navigation (Medtronic, Louisville, CO,
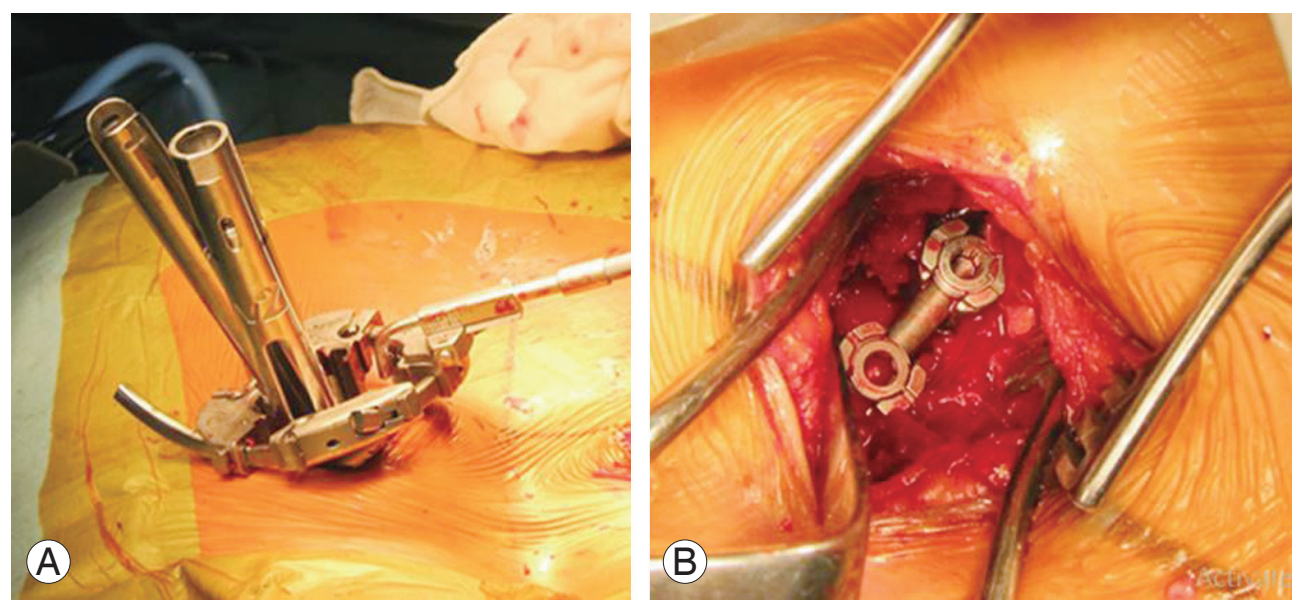

Fig. 3. (A, B) MIS-PLF via the mini-open Wiltse approach using a pipeline expandable retractor. Most MIS-PLF surgeries were performed by the technique described by Kotani. The above mentioned technique was only applied to the isthmic spondylolisthesis case (only one case). MIS-PLF, minimally invasive surgical-posterolateral fusion. 

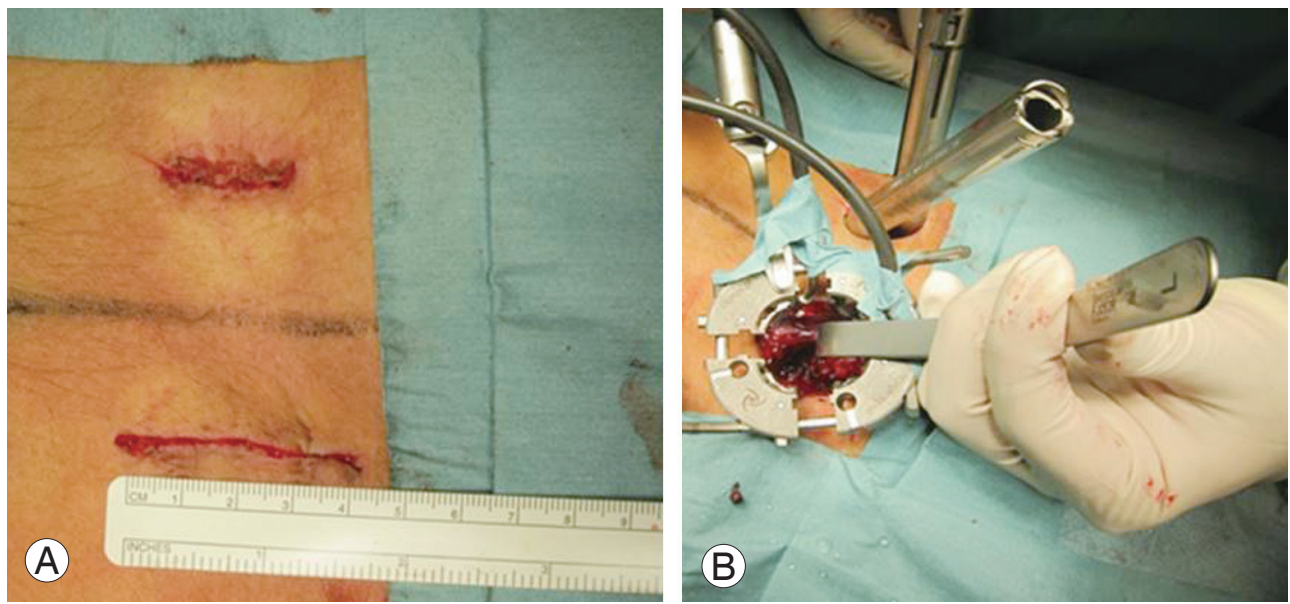

Fig. 4. (A, B) Minimally invasive surgical-transforaminal lumbar interbody fusion via the unilateral mini-open Wiltse approach and percutaneous pedicle screws on the other side.

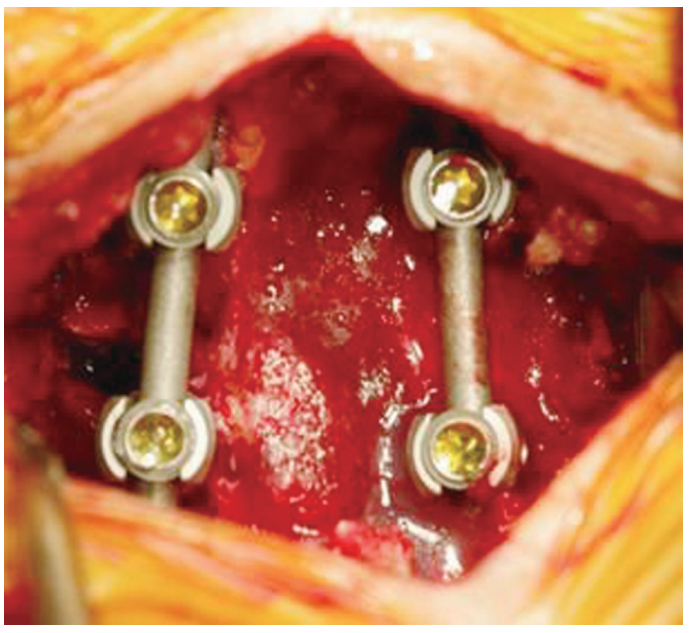

Fig. 5. Midline lumbar fusion via a small, single, midline incision with modified cortical bone trajectory.

USA). MIDLF was performed with a small, single posterior midline incision $(3.5 \mathrm{~cm})$. After the exposure of the posterior elements within the lateral margin of the facet joints, mCBT screws were inserted with $\mathrm{O}$-arm navigation (Medtronic). The screws have a large diameter $(>5.5$ $\mathrm{mm}$ ) and are inserted to engage the anterolateral part of the vertebral body. The orientation of the mCBT screws allows their insertion through the small incision without the need for dissection beyond the facet joints (Fig. 5). The entry point was created using high-speed burrs, followed by the insertion of a pedicle finder and tapping. Finally, the mCBT screws were applied. With the distraction applied between the mCBT screws, TLIF was performed with a PEEK cage packed with autologous bone graft and hydroxyapatite granules.

\section{Statistical analysis}

Statistical analyses were performed using one-way analysis of variance to compare the differences between the continuous variables of each group. The conservative post hoc testing method was used to identify specific group interactions. Chi-square test was used to analyze the categorical variables. Statistical analyses were performed using $\mathrm{R}$ ver. 3.4 (https://www.r-project.org/). All tests with a significance level of $p<0.05$ were considered statistically significant.

\section{Results}

Overall follow-up was performed for $92 \%$ of the cases; five patients were lost to follow-up (two in the MIS-PLF group, one in the MIS-TLIF group, and two in the MIDLF group). There was no significant difference in the sex distribution or BMI between the groups. The mean age of the patients in the MIS-TLIF group was significantly lower than that of the patients in the other groups.

The mean operation time was significantly shorter in the MIDLF group ( $111 \pm 23.98$ minutes) than in the MISPLF (132 \pm 25.08 minutes) and MIS-TLIF groups (167 \pm 20.6 minutes). The mean bleeding amount was significantly lower in the MIDLF group $(112.5 \pm 85.2 \mathrm{~mL})$ than in the MIS-PLF $(245 \pm 162.11 \mathrm{~mL})$ and MIS-TLIF groups $(283.7 \pm 104.66 \mathrm{~mL})$.

The mean CRP values were significantly lower in 
the MIDLF group $(1.38 \pm 1.2 \mathrm{mg} / \mathrm{L})$ at POD1 than in the MIS-PLF $(3.24 \pm 2.82 \mathrm{mg} / \mathrm{L})$ and MIS-TLIF groups $(2.07 \pm 0.88 \mathrm{mg} / \mathrm{L})$; however, there was no significant difference between the three groups in terms of the mean CRP values at POD7. The mean CK values were significantly lower in the MIDLF group (POD1, 377 $\pm 584 \mathrm{U} /$ L; POD7, 66.86 $\pm 32.4 \mathrm{U} / \mathrm{L}$ ) than in the MIS-PLF (POD1, $606.38 \pm 326.71 \mathrm{U} / \mathrm{L}$; POD7, $161.59 \pm 147.21 \mathrm{U} / \mathrm{L})$ and MIS-TLIF groups (POD1, 780.33 $\pm 458.74 \mathrm{U} / \mathrm{L}$; POD7,
$117.73 \pm 57.72 \mathrm{U} / \mathrm{L})$.

All the groups showed significant improvements in the postoperative JOABPEQ score, VAS score, and ODI, whereas there was no significant difference between the groups. Clinical outcome scores at the 1-year follow-up are listed in Table 2.

MIDLF resulted in a greater increase in the LLA and SDA postoperatively. The mean increase in the LLA was $3.34^{\circ} \pm 8.23^{\circ}$ for the MIS-PLF group, $3.17^{\circ} \pm 4.47^{\circ}$ for the

Table 2. Clinical outcome scores at the 1-year follow-up

\begin{tabular}{|c|c|c|c|c|c|c|c|c|c|}
\hline \multirow{2}{*}{ Variable } & \multicolumn{3}{|c|}{ MIS-posterolateral fusion } & \multicolumn{3}{|c|}{$\begin{array}{l}\text { MIS-transforaminal lumbar } \\
\text { interbody fusion }\end{array}$} & \multicolumn{3}{|c|}{ Midline lumbar fusion } \\
\hline & Preop & Postop & $p$-value & Preop & Postop & $p$-value & Preop & Postop & $p$-value \\
\hline \multicolumn{10}{|c|}{$\begin{array}{l}\text { Japanese orthopedic association back } \\
\text { pain evaluation questionnaire }\end{array}$} \\
\hline Low back pain & $56.3 \pm 7.6$ & $78 \pm 9.9$ & $<0.001$ & $45.9 \pm 9.8$ & $80.4 \pm 7.3$ & $<0.001$ & $45.5 \pm 7.4$ & $79.6 \pm 9.2$ & $<0.001$ \\
\hline Walking ability & $43.9 \pm 9.5$ & $78.5 \pm 8$ & $<0.001$ & $43.6 \pm 9.5$ & $75.5 \pm 8.5$ & $<0.001$ & $41.5 \pm 6.4$ & $79.5 \pm 8$ & $<0.001$ \\
\hline Lumbar function & $49 \pm 14.2$ & $77.2 \pm 16$ & $<0.001$ & $52.7 \pm 2.1$ & $79 \pm 19.5$ & $<0.001$ & $48.8 \pm 11.1$ & $78.9 \pm 7.3$ & $<0.001$ \\
\hline Social life & $32.6 \pm 3.6$ & $56 \pm 7.1$ & $<0.001$ & $32.8 \pm 4.1$ & $57.6 \pm 4.8$ & $<0.001$ & $33.6 \pm 5.22$ & $58.5 \pm 5.8$ & $<0.001$ \\
\hline Mental health & $36.6 \pm 3.8$ & $61.8 \pm 6.9$ & $<0.001$ & $34.8 \pm 4.1$ & $65 \pm 4.2$ & $<0.001$ & $37.6 \pm 5.5$ & $67.5 \pm 5.8$ & $<0.001$ \\
\hline \multicolumn{10}{|l|}{ Visual Analog Scale } \\
\hline Low back pain & $44.6 \pm 6.8$ & $18.5 \pm 3.8$ & $<0.001$ & $40.3 \pm 3.9$ & $19.3 \pm 3.7$ & $<0.001$ & $40.1 \pm 6.4$ & $17.2 \pm 3.4$ & $<0.001$ \\
\hline Leg pain & $50.5 \pm 5.8$ & $15.8 \pm 3.2$ & $<0.001$ & $49.3 \pm 8.3$ & $17.2 \pm 5.6$ & $<0.001$ & $50.1 \pm 5.6$ & $14 \pm 4.2$ & $<0.001$ \\
\hline Oswestry Disability Index & $58.1 \pm 6.3$ & $15.5 \pm 4.9$ & $<0.001$ & $55.7 \pm 6.2$ & $17.1 \pm 5.2$ & $<0.001$ & $57.5 \pm 2.3$ & $17.3 \pm 2.7$ & $<0.001$ \\
\hline
\end{tabular}

Values are presented as mean \pm standard deviation.

MIS, minimally invasive surgical; Preop, preoperative; Postop, postoperative.
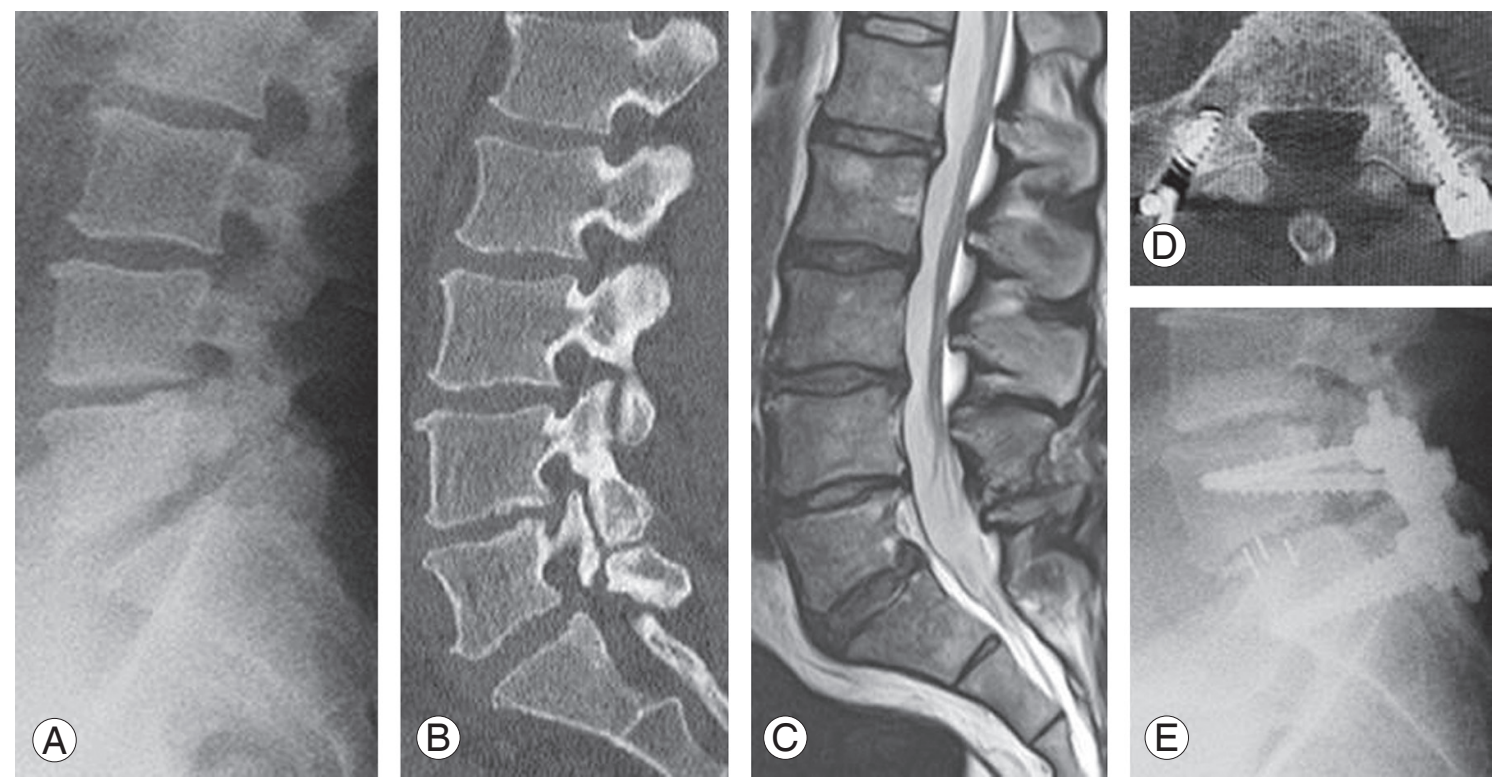

Fig. 6. (A-E) L5-S1 minimally invasive surgical-transforaminal lumbar interbody fusion for L5 isthmic spondylolisthesis. 
MIS-TLIF group, and $7.9^{\circ} \pm 11.8^{\circ}$ for the MIDLF group. However, the mean increase in the SDA was $2.25^{\circ} \pm 4.42^{\circ}$, $1.38^{\circ} \pm 4.69^{\circ}$, and $3.64^{\circ} \pm 2.7^{\circ}$ for each group, respectively.
There was no significant difference between the three groups in terms of restoration of anterior $\mathrm{DH}$, whereas the MIDLF $(2.8 \pm 2.5 \mathrm{~mm})$ and MIS-TLIF groups $(2.73 \pm 2.85$
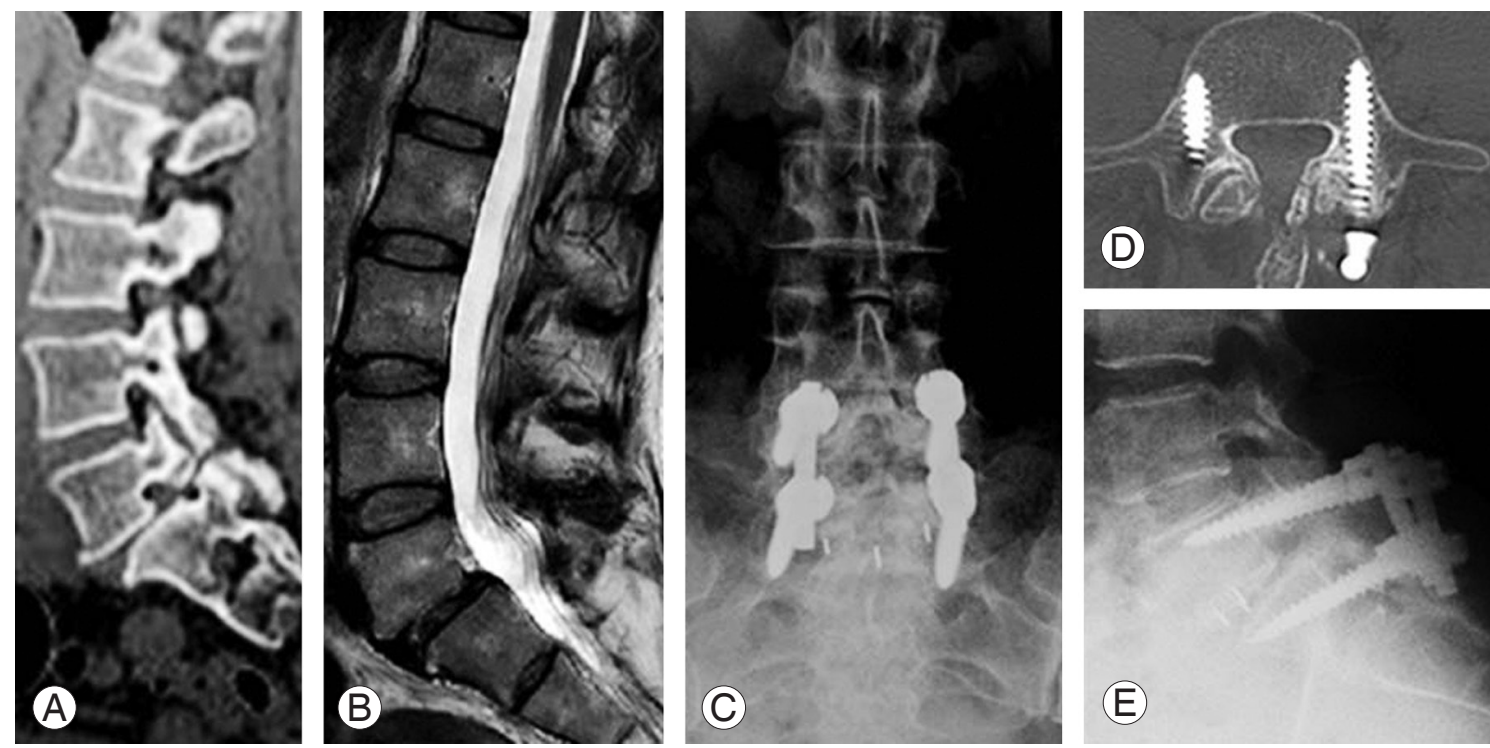

Fig. 7. (A-E) L5-S1 midline lumbar fusion with modified cortical bone trajectory for L5 isthmic spondylolisthesis.

Table 3. Operative and radiological results

\begin{tabular}{|c|c|c|c|c|}
\hline Variable & $\begin{array}{l}\text { MIS-posterolateral } \\
\text { fusion }\end{array}$ & $\begin{array}{l}\text { MIS-transforaminal lumbar } \\
\text { interbody fusion }\end{array}$ & $\begin{array}{l}\text { Midline lumbar } \\
\text { fusion }\end{array}$ & $p$-value \\
\hline Mean operation time (min) & $132 \pm 25.08$ & $167 \pm 20.6$ & $111 \pm 23.98^{*}$ & 0.001 \\
\hline Mean bleeding amount (mL) & $245 \pm 162.11$ & $283.7 \pm 104.66$ & $112.5 \pm 85.2^{*}$ & 0.001 \\
\hline \multicolumn{5}{|l|}{ Mean C-reactive protein value (mg/dL) } \\
\hline POD1 & $3.24 \pm 2.82$ & $2.07 \pm 0.88$ & $1.38 \pm 1.2^{*}$ & 0.009 \\
\hline POD7 & $1.92 \pm 1.1$ & $1.90 \pm 2.38$ & $1.35 \pm 1.25$ & 0.42 \\
\hline \multicolumn{5}{|l|}{ Mean creatine kinase value (U/L) } \\
\hline POD1 & $606.38 \pm 326.71$ & $780.33 \pm 458.74$ & $377 \pm 584^{*}$ & 0.04 \\
\hline POD7 & $161.59 \pm 147.21$ & $117.73 \pm 57.72$ & $66.86 \pm 32.4^{*}$ & 0.008 \\
\hline Mean increase of lumbar lordosis angle $\left({ }^{\circ}\right)$ & $3.34 \pm 8.23$ & $3.17 \pm 4.47$ & $7.9 \pm 11.8$ & 0.17 \\
\hline Mean increase of segmental disc angle $\left({ }^{\circ}\right)$ & $2.25 \pm 4.42$ & $1.38 \pm 4.69$ & $3.64 \pm 2.7$ & 0.22 \\
\hline \multicolumn{5}{|l|}{ Mean increase of disc height (mm) } \\
\hline Anterior & $1.50 \pm 3.78$ & $2.75 \pm 2.88$ & $2.7 \pm 3.3$ & 0.06 \\
\hline Middle & $0.28 \pm 1.99$ & $2.73 \pm 2.85^{*}$ & $2.8 \pm 2.5^{*}$ & 0.02 \\
\hline Posterior & $0.33 \pm 1.95$ & $0.17 \pm 1.7$ & $1.19 \pm 2.65$ & 0.07 \\
\hline Slippage reduction (\%) & $12.4 \pm 1.8$ & $14.2 \pm 2.3$ & $16.5 \pm 1.5$ & 0.06 \\
\hline Loss of correction $(\%)$ & $5.2 \pm 0.9$ & $4.2 \pm 1.1$ & $2.6 \pm 0.8$ & 0.3 \\
\hline Fusion rate $(\%)$ & 90 & 100 & 100 & 0.95 \\
\hline Incidence of screw loosening (\%) & 10 (2 cases) & 7.14 (1 case) & 4.76 (1 case) & 0.83 \\
\hline
\end{tabular}

Values are presented as mean \pm standard deviation, unless otherwise stated.

MIS, minimally invasive surgical; POD, postoperative day.

" $p<0.05$. 
$\mathrm{mm}$ ) showed a significant increase in the middle $\mathrm{DH}$ compared with that observed in the MIS-PLF group $(0.28 \pm 1.99 \mathrm{~mm})$. Moreover, the MIDLF group showed a greater increase in the posterior DH $(1.19 \pm 2.65 \mathrm{~mm})$ compared with that observed in the MIS-PLF $(0.33 \pm 1.95$ $\mathrm{mm})$ and MIS-TLIF groups $(0.17 \pm 1.7 \mathrm{~mm})$.

The percentage loss of correction at 6 months postoperatively was lower in the MIDLF group (2.6\%) than in the MIS-PLF (5.2\%) and MIS-TLIF (4.2\%) groups. The fusion rate was $100 \%$ in the MIDLF and MIS-TLIF groups (Figs. $6,7)$, whereas it was $90 \%$ in the MIS-PLF group, with two cases of nonunion. The incidence of screw loosening was lower in the MIDLF group (4.76\%) than in the MIS-PLF (10\%) and MIS-TLIF (7.14\%) groups. One patient of the MIS-PLF group who had nonunion underwent revision after 1 year using MIDLF. Another patient with L5-S1 IS in the MIS-PLF group developed pseudoarthrosis, which was revised by extending the fusion to L4-S2. S1 pedicular screw malposition was revised immediately intraoperatively in the MIS-PLF group. One patient with L4-L5 DS in the MIS-TLIF group had L3-L4 decompression one week postoperatively. The operative and radiological results are shown in Table 3.

\section{Discussion}

Recently, considerable advances have been made in MIS techniques for lumbar spine fusion, with the development of intraoperative imaging facilities and retraction systems. MIS techniques are considered in the management of different spinal pathologies $[8,11]$. Many publications have reported the advantages of these techniques over open conventional methods, and early results were favorable $[11,12,14,18]$.

Kotani et al. [14] reported better results with MIS-PLF than with PLF in terms of reduction in low back pain and improvement in the clinical outcomes of the patients, with reduction in intraoperative and postoperative blood loss and a comparable fusion rate in both the groups. Many authors have reported superior results of MIS-TLIF compared with those of conventional open TLIF in terms of functional outcome, postoperative pain, intraoperative blood loss, postoperative blood transfusion, and hospital stay. There was no significant difference in the fusion rates of both groups $[1,18]$.

However, thus far, few studies have compared these different MIS techniques. In the present study, we compared the clinical and radiological outcomes of MIS-PLF, MISTLIF, and MIDLF for the management of single-level lumbar IS and DS.

MIDLF is a recent MIS fusion technique that comprises transforaminal interbody arthrodesis and posterior instrumentation using CBT screws [20]. The use of CBT screws is a recent technique of posterior lumbar spinal instrumentation that allows the engagement of the screws to the hard cortical bone of the lamina with better anchoring than that using traditional pedicular screws. Being inserted in a divergent trajectory, CBT screws do not require dissection beyond the facet joints for their insertions $[19,20]$.

Matsukawa et al. [21] conducted a biomechanical study and reported higher insertion torque of CBT screws than that of traditional screws. It may be particularly important when performing lumbar fusion in older patients with poor bone density.

The original CBT technique utilized small and short screws with a high incidence of screw breakage. Moreover, they lacked anterior load sharing. Therefore, we replace the original CBT screws by the modified CBT screws that are longer, have a larger diameter, and provide superior engagement with the anterior vertebral body.

In the present study, MIS-TLIF showed significantly less satisfactory results in terms of intraoperative bleeding amount and operation time than that observed with MIS-PLF and MIDLF owing to limited access to the interbody space, which required more soft tissue dissection and invasiveness. Peng et al. [22] and Shunwu et al. [23] reported longer operation time with MIS-TLIF because of a narrow field of view and difficult access to the disc space in addition to the high technical skill required for this technique, wherein the surgeon should be familiar with the approach and anatomical landmarks.

The advantage of MIS-PLF and MIS-TLIF via the miniopen Wiltse approach is the absence of extensive muscle dissection because these techniques can be performed via the muscular plane between the multifidus and longissimus muscles with less muscle injury than conventional PLF and TLIF. While the drawbacks of MIS-PLF and MIS-TLIF were the narrow field of view, use of only twodimensional image, and a significant learning curve at that time that complicated the procedure, resulting in a longer operation time and more intraoperative bleeding, particularly for MIS-TLIF. Lack of cortical bone support and low pullout strength of the traditional pedicular screw in 
the MIS-PLF and MIS-TLIF techniques led to the higher incidence of screw loosening and higher loss of correction at 6 months postoperatively. However, overall, the interbody fusion methods (MIS-TLIF and MIDLF) showed a significantly better fusion rate and were significantly more effective in restoring middle DH than MIS-PLF.

MIDLF could be performed through a small midline incision with minimal soft tissue injury. It was associated with a significantly shorter operation time and significantly lower values of soft tissue invasiveness parameters (CRP and CK). The orientation of mCBT screw insertion did not require dissection beyond the facet joints. This reduced the damage to the posterior ramus and resulted in significantly less bleeding than with MIS-PLF and MISTLIF. The higher insertion torque and pullout strength of mCBT screws used in MIDLF was associated with less screw loosening and loss of correction at 6 months postoperatively and a higher fusion rate. Mizuno et al. [20] reported the clinical and radiological outcomes of MIDLF using CBT with better recovery of the postoperative inflammatory markers due to its minimal invasiveness. Further, it allows fusion, decompression, and instrumentation through the same approach. (20)The interbody fusion together with $\mathrm{mCBT}$ made MIDLF more effective in restoring the LLA, SDA, and DH than MIS-PLF and MIS-TLIF. The relatively fewer number of cases in the MIS-PLF and MIS-TLIF groups was a study limitation. Further, it was not a randomized controlled study. The learning curve of these high technically demanding techniques may have influenced the outcomes. The inclusion of IS and DS cases in the same study was another limitation. More studies that analyze the outcomes of different MIS fusion techniques are required.

\section{Conclusions}

The clinical and radiological performances of the three types of MIS fusion techniques in the management of lumbar IS and DS were compared. There was no significant difference between the three groups in terms of fusion, screw loosening, correction of slippage, loss of correction, and correction of sagittal alignment. MIDLF provided less invasiveness with significantly shorter operation time, less bleeding amount, and lower values of invasiveness markers. This could be attributed to the single, small midline incision of MIDLF with easier access to the disc space than with the narrow access and greater muscle retraction of the mini-open Wiltse approach of MIS-TLIF and MIS-PLF.

\section{Conflict of Interest}

No potential conflict of interest relevant to this article was reported.

\section{Author Contributions}

ME: conception and design, drafting of the manuscript, and analysis of data; EEM: drafting of the manuscript and supervision; YR: drafting of the manuscript and supervision; AET: critical revision; IS: data acquisition and analysis of data; RF: data acquisition and analysis of data; and YK: conception and design, drafting of the manuscript, and final approval of the version to be published.

\section{References}

1. Lee KH, Yue WM, Yeo W, Soeharno H, Tan SB. Clinical and radiological outcomes of open versus minimally invasive transforaminal lumbar interbody fusion. Eur Spine J 2012;21:2265-70.

2. Sethi A, Lee S, Vaidya R. Transforaminal lumbar interbody fusion using unilateral pedicle screws and a translaminar screw. Eur Spine J 2009;18:430-4.

3. Gibson JN, Grant IC, Waddell G. The Cochrane review of surgery for lumbar disc prolapse and degenerative lumbar spondylosis. Spine (Phila Pa 1976) 1999;24:1820-32.

4. Fritzell P, Hagg O, Wessberg P, Nordwall A; Swedish Lumbar Spine Study Group. Chronic low back pain and fusion: a comparison of three surgical techniques: a prospective multicenter randomized study from the Swedish lumbar spine study group. Spine (Phila Pa 1976) 2002;27:1131-41.

5. Elias WJ, Simmons NE, Kaptain GJ, Chadduck JB, Whitehill R. Complications of posterior lumbar interbody fusion when using a titanium threaded cage device. J Neurosurg 2000;93(1 Suppl):45-52.

6. Benglis DM, Elhammady MS, Levi AD, Vanni S. Minimally invasive anterolateral approaches for the treatment of back pain and adult degenerative deformity. Neurosurgery 2008;63(3 Suppl):191-6.

7. Mummaneni PV, Tu TH, Ziewacz JE, Akinbo OC, Deviren V, Mundis GM. The role of minimally inva- 
sive techniques in the treatment of adult spinal deformity. Neurosurg Clin N Am 2013;24:231-48.

8. Khan NR, Clark AJ, Lee SL, Venable GT, Rossi NB, Foley KT. Surgical outcomes for minimally invasive vs open transforaminal lumbar interbody fusion: an updated systematic review and meta-analysis. Neurosurgery 2015;77:847-74.

9. Snyder LA, O’Toole J, Eichholz KM, Perez-Cruet MJ, Fessler R. The technological development of minimally invasive spine surgery. Biomed Res Int 2014;2014:293582.

10. Fessler RG, O’Toole JE, Eichholz KM, Perez-Cruet MJ. The development of minimally invasive spine surgery. Neurosurg Clin N Am 2006;17:401-9.

11. Rodriguez-Vela J, Lobo-Escolar A, Joven E, MunozMarin J, Herrera A, Velilla J. Clinical outcomes of minimally invasive versus open approach for onelevel transforaminal lumbar interbody fusion at the 3- to 4-year follow-up. Eur Spine J 2013;22:2857-63.

12. Tsutsumimoto $\mathrm{T}$, Shimogata M, Ohta H, Misawa H. Mini-open versus conventional open posterior lumbar interbody fusion for the treatment of lumbar degenerative spondylolisthesis: comparison of paraspinal muscle damage and slip reduction. Spine (Phila Pa 1976) 2009;34:1923-8.

13. Forsth P, Michaelsson K, Sanden B. Does fusion improve the outcome after decompressive surgery for lumbar spinal stenosis?: a two-year follow-up study involving 5390 patients. Bone Joint J 2013;95-B:9605.

14. Kotani Y, Abumi K, Ito M, Sudo H, Abe Y, Minami A. Mid-term clinical results of minimally invasive decompression and posterolateral fusion with percutaneous pedicle screws versus conventional approach for degenerative spondylolisthesis with spinal stenosis. Eur Spine J 2012;21:1171-7.
15. Lowe TG, Tahernia AD, O’Brien MF, Smith DA. Unilateral transforaminal posterior lumbar interbody fusion (TLIF): indications, technique, and 2-year results. J Spinal Disord Tech 2002;15:31-8.

16. Rantanen J, Hurme M, Falck B, et al. The lumbar multifidus muscle five years after surgery for a lumbar intervertebral disc herniation. Spine (Phila $\mathrm{Pa}$ 1976) 1993;18:568-74.

17. Lv Y, Chen J, Chen J, et al. Three-year postoperative outcomes between MIS and conventional TLIF in 1-segment lumbar disc herniation. Minim Invasive Ther Allied Technol 2017;26:168-76.

18. Phan K, Rao PJ, Kam AC, Mobbs RJ. Minimally invasive versus open transforaminal lumbar interbody fusion for treatment of degenerative lumbar disease: systematic review and meta-analysis. Eur Spine J 2015;24:1017-30.

19. Santoni BG, Hynes RA, McGilvray KC, et al. Cortical bone trajectory for lumbar pedicle screws. Spine J 2009;9:366-73.

20. Mizuno M, Kuraishi K, Umeda Y, Sano T, Tsuji M, Suzuki H. Midline lumbar fusion with cortical bone trajectory screw. Neurol Med Chir (Tokyo) 2014;54:716-21.

21. Matsukawa K, Yato Y, Nemoto O, Imabayashi H, Asazuma T, Nemoto K. Morphometric measurement of cortical bone trajectory for lumbar pedicle screw insertion using computed tomography. J Spinal Disord Tech 2013;26:E248-53.

22. Peng CW, Yue WM, Poh SY, Yeo W, Tan SB. Clinical and radiological outcomes of minimally invasive versus open transforaminal lumbar interbody fusion. Spine (Phila Pa 1976) 2009;34:1385-9.

23. Shunwu F, Xing Z, Fengdong Z, Xiangqian F. Minimally invasive transforaminal lumbar interbody fusion for the treatment of degenerative lumbar diseases. Spine (Phila Pa 1976) 2010;35:1615-20. 